\section{Zumbido em paciente com megadolicobasilar: relato de caso}

Fransérgio E. M. Cavallari', Maria Cristina L. C.

\section{Tinnitus in a patient with dolichomegabasilar: a case report}

Feres $^{2}$, Miriam L Isac ${ }^{3}$,José A. A. Oliveira ${ }^{4}$

\section{Resumo}

zumbido pode se manifestar como sintoma de diversas doenças, que podem ser congênitas, infecciosas, neoplásicas, neurológicas, traumáticas, metabólicas, vasculares e mistas. Apresenta-se com intensidade variável, e em sua forma mais severa pode ser altamente incomodativo e angustiante. A investigação do diagnóstico diferencial nos casos de zumbido deve ser cuidadosamente conduzida, com base em diferentes tipos de exames complementares, como a audiometria tonal liminar, imitanciometria, audiometria de respostas evocadas de tronco cerebral e exames de imagem, principalmente a ressonância nuclear magnética. Neste artigo, mostraremos em um caso de zumbido secundário a rara alteração anatômica da artéria basilar, chamada megadolicobasilar, causando compressão do VIII par craniano, para salientar a importância dos exames complementares na investigação e conduta do paciente com zumbido.

\section{Palavras-chave: zumbido, megadolicobasilar, compressão vascular.}

Key words: tinnitus, dolichomegabasilar, vascular compression.

\begin{abstract}
T
innitus may appear as a symptom in a large number of pathologies, like congenital, infectious, neoplastical, neurological, traumatic, metabolical, vascular or mixed. It is presented with variable intensity, and, in its most severe form, may even undergo patient into suicide. Therefore, the differential diagnosis must be done carefully, based on different kinds of complementary tests like pure tone audiometry, imitanciometry, auditory brainstem responses and nuclear magnetic resonance. In this article, we show a case of tinnitus secondary to a rare anatomic alteration of the basilar artery, named dolichomegabasilar, leading to compression of the eighth cranial nerve, to emphasize the importance of the complementary tests on investigation and management of the tinnitus patient.
\end{abstract}

${ }^{1}$ Médico Residente da disciplina de Otorrinolaringologia.

${ }^{2}$ Professora Doutora da disciplina de Otorrinolaringologia, coordenadora do Ambulatório de Investigação e Reabilitação Auditiva.

${ }^{3}$ Médica Assistente da disciplina de Otorrinolaringologia, responsável pelo Laboratório de Eletrofisiologia da Audição. ${ }^{4}$ Professor Titular, chefe da Disciplina de Otorrinolaringologia.

Instituição: Faculdade de Medicina de Ribeirão Preto da Universidade de São Paulo.

Endereço para Correspondência: Av. Bandeirantes, $390012^{\circ}$ andar Ribeirão Preto SP14090-900.

Fax (0xx16)602-2860 - E-mail: mariacristina@roo.fmrp.usp.br

Artigo recebido em 02 de outubro de 2002. Artigo aceito em 29 de novembro de 2002. 


\section{INTRODUÇÃO}

O zumbido pode ser definido como a percepção de um ruído sem detecção de fonte sonora correspondente, podendo ser uni ou bilateral ${ }^{1}$. Pode apresentar-se com origens diversas e de intensidade variável, chegando, em sua forma severa, a ser altamente incapacitante e até mesmo levar um paciente à atitude extrema de tentar suicídio ${ }^{2}$. É percebido na maioria das vezes apenas pelo paciente, quando é chamado de subjetivo. Nas vezes em que um observador pode percebê-lo, é denominado objetivo.

Várias lesões ou enfermidades podem levar à manifestação do zumbido, como doenças congênitas, infecciosas, neoplásicas, neurológicas, traumáticas, metabólicas, mistas e vasculares ${ }^{3}$.

No diagnóstico diferencial do zumbido, além de uma anamnese e exame físico que identifiquem ou excluam as doenças citadas acima, podemos lançar mão de exames complementares como a audiometria tonal liminar com imitanciometria $(\mathrm{A}+\mathrm{I})$, e, principalmente em casos em que a queixa do paciente ou o resultado da $\mathrm{A}+\mathrm{I}$ mostram assimetria, fazemos o Potencial Evocado Auditivo de Tronco Cerebral (BERA - Brainstem Evoked Responses Audiometry). Na presença de alterações, exames de imagem se fazem necessários posteriormente, sendo a Ressonância Nuclear Magnética o exame mais apropriado para esta investigação.

O BERA é muito importante na diferenciação da perda auditiva central ou periférica, e na detecção de tumores de tronco cerebral e do neurinoma do acústicón.

O objetivo deste relato é mostrar o caso de um paciente com zumbido unilateral, submetido aos métodos de investigação descritos acima, de forma seqüencial, levando-nos ao achado de uma alteração anatômica rara, porém já descrita como desencadeante de sintomas vestibulococleares.

\section{RELATO DO CASO}

V.M.A., 66 anos, sexo masculino, branco, procedente de Ribeirão Preto-SP, procurou o serviço de Otorrinolaringologia do Hospital das Clínicas da Faculdade de Medicina de Ribeirão Preto da USP, encaminhado pelo Ambulatório de Cefaléia deste mesmo serviço, onde era seguido por um quadro de migrânea sem aura, com queixa de zumbido há 6 anos. Relatava zumbido contínuo à esquerda, de instalação insidiosa, sem fatores acompanhantes, pior quando concomitante com cefaléia holocraniana.

Negava disacusia no início do quadro, porém na ocasião do atendimento otorrinolaringológico apresentava queixa de leve hipoacusia bilateral, mais intensa à esquerda. Negava tontura.

Não fez tratamento prévio para o zumbido. Já havia feito uso de diversos analgésicos para tratamento da cefaléia.
Usava na época do primeiro atendimento as seguintes medicações: clorpromazina $25 \mathrm{mg} /$ dia e amitriptilina $25 \mathrm{mg}$ $12 / 12 \mathrm{~h}$.

Negava hipertensão arterial sistêmica, diabetes mellitus ou qualquer tipo de cardiopatia.

Ao exame físico, apresentava otoscopia normal bilateralmente, sem nistagmo espontâneo ou semiespontâneo, com exame do aparelho vestibular e dos pares cranianos normais.

Foi submetido a audiometria tonal liminar (Quadro 1), onde apresentou perda neurossensorial de grau leve a moderado, com exame normal em graves. Posteriormente, foi submetido a um BERA (Quadro 3), que mostrou alargamento de intervalo entre as ondas I e III à esquerda. Mediante esta alteração, foi solicitada uma Ressonância Nuclear Magnética de encéfalo com ênfase no oitavo par craneano, a qual evidenciou ectasia de artéria basilar à esquerda (megadolicobasilar), causando compressão do nervo em questão (Figura 1).

Quadro 1. Exame de Audiometria Tonal Liminar.

\begin{tabular}{|ccc|}
\hline & \multicolumn{2}{c|}{ Limiar audiométrico $(\mathrm{dB})$} \\
Freqüência $(\mathrm{Hz})$ & Ouvido Direito & Ouvido Esquerdo \\
\hline 250 & 25 & 35 \\
500 & 15 & 25 \\
1000 & 15 & 25 \\
2000 & 40 & 50 \\
3000 & 45 & 55 \\
4000 & 45 & 50 \\
8000 & 20 & 50 \\
\hline
\end{tabular}

Quadro 2. Índice Percentual de Reconhecimento de Fala

\begin{tabular}{|ccc|}
\hline & Ouvido Direito & Ouvido Esquerdo \\
\hline Intensidade (dB) & 65 & 75 \\
\% IPRF & 96 & 92 \\
\hline
\end{tabular}

Quadro 3. Resultados do BERA.

\begin{tabular}{|lcc|}
\hline & Ouvido Direito & Ouvido Esquerdo \\
\hline Latência Onda I & $1,74 \mathrm{~ms}$ & $1,52 \mathrm{~ms}$ \\
Latência Onda III & $3,86 \mathrm{~ms}$ & $4,16 \mathrm{~ms}$ \\
Latência Onda V & $5,92 \mathrm{~ms}$ & $6,02 \mathrm{~ms}$ \\
Amplitude Onda I & $0,34 \mu \mathrm{V}$ & $0,42 \mu \mathrm{V}$ \\
Amplitude Onda V & $0,67 \mu \mathrm{V}$ & $0,41 \mu \mathrm{V}$ \\
Intervalo I-III & $2,12 \mathrm{~ms}$ & $2,64 \mathrm{~ms}$ \\
Intervalo III-V & $2,06 \mathrm{~ms}$ & $1,86 \mathrm{~ms}$ \\
Intervalo I-V & $4,18 \mathrm{~ms}$ & $4,50 \mathrm{~ms}$ \\
\hline
\end{tabular}


O paciente foi então medicado com clonazepan 2,5 $\mathrm{mg} / \mathrm{ml}, 5$ gotas ao dia, orientado a utilizar-se de mascaramento e encaminhado ao serviço de Neurocirurgia para verificar possibilidade de tratamento cirúrgico. Após avaliação cuidadosa pelo setor, a equipe de Neurocirurgia descartou a ocorrência de formação aneurismática, permanecendo o diagnóstico de anomalia anatômica benigna, o que não indicou tratamento cirúrgico. O paciente permanece em seguimento, com bom controle clínico do sintoma.

\section{DISCUSSÃO}

O zumbido unilateral é um sintoma que chama muito a atenção do otorrinolaringologista por ser um sinal possivelmente retrococlear e ter relação importante com patologias tumorais, como o neurinoma do VIII par. Comumente, não há alteração do paciente ao exame físico, e uma investigação complementar sempre se faz necessária.

Os pacientes com zumbido apresentam alterações associadas com lentificação nas latências interpicos das ondas O-I e I-V no BERA, do lado afetado, mostrando redução significativa na amplitude das ondas I e III, e às vezes na onda V. O exame nos permite, assim, aferir indiretamente o distúrbio provocado pelo zumbido. Em estudos de LavernheLemaire e Beutter (1989 e 1997) ${ }^{4}$ observou-se que pacientes com latência I-V homolateral ao zumbido maior que a latência contralateral, o sintoma deve ter origem central, enquanto nos pacientes com o zumbido de origem periférica, a latência homolateral I-V é menor que a latência I-V contralateral. Essas alterações são mostradas independentemente do tipo ou da freqüência do zumbido. Finalmente, podemos dizer
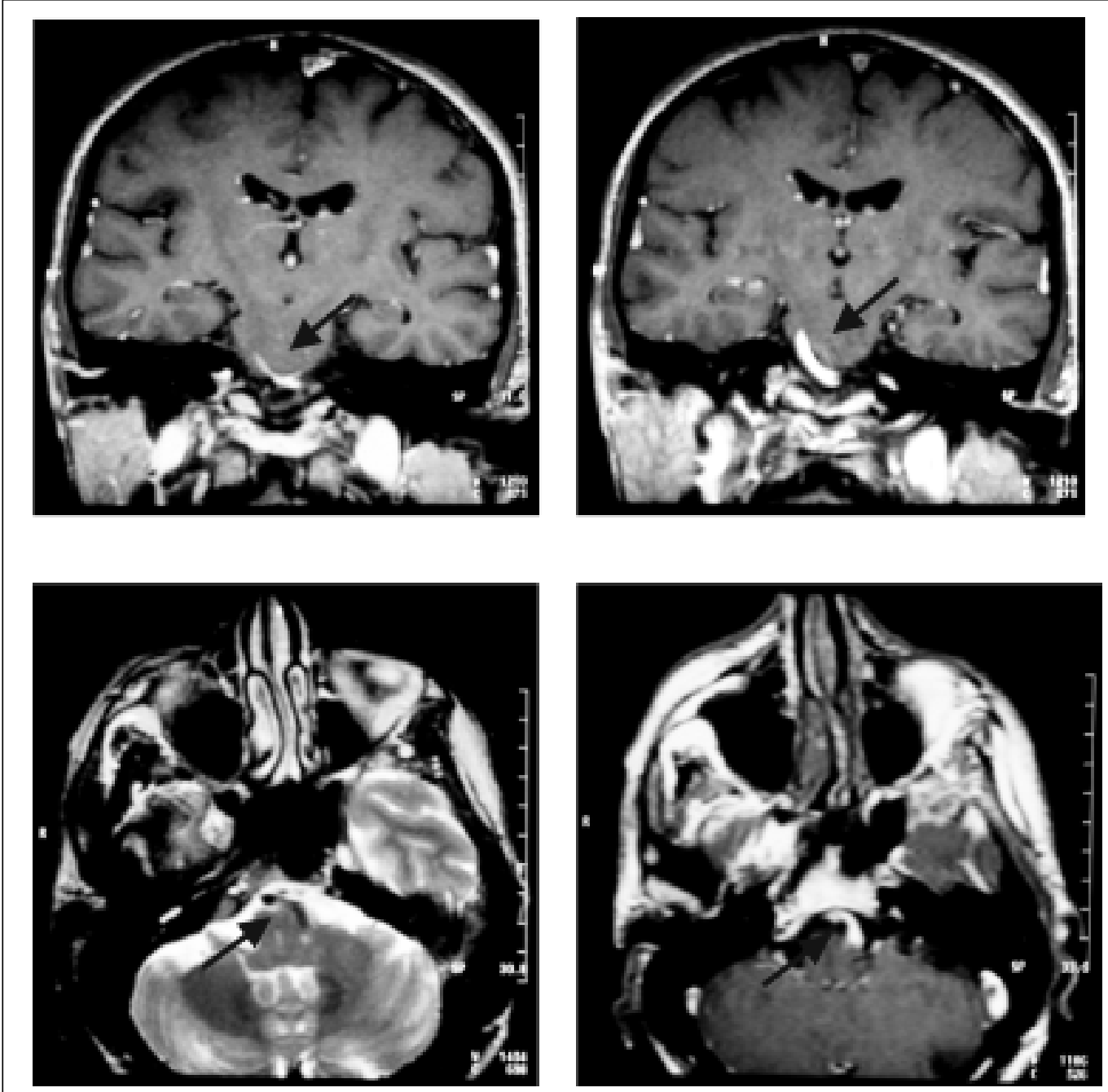

Figura 1. imagem da ectasia tortuosa da artéria basilar, em duas diferentes incidências do exame de ressonância nuclear magnética. As setas apontam para a alteração. 
que as alterações de ondas I e III, principalmente, sugerem fortemente envolvimento dos sistemas eferentes.

Em casos como o deste paciente, de posse dos dados do BERA, devemos fazer um exame de imagem para localizar o problema no sistema nervoso, sendo a mais indicada a RNM, e, quando possível no caso de suspeita de lesão vascular como razão do zumbido, angiorresonância magnética.

A megadolicobasilar é uma alteração rara, muitas vezes assintomática, que pode causar uma enorme gama de sintomas ${ }^{5,6}$. Já foram descritos casos de surdez súbita, zumbido, vertigem, neuralgia do trigêmeo, paralisia ou espasmo facial. São casos raros, porém é essencial que se faça o diagnóstico diferencial, pois os sintomas serão refratários ao tratamento clínico na maioria das vezes. O diagnóstico é feito pela ressonância ou angiorresonância magnética ${ }^{5,7}$. O tratamento pode ser cirúrgico, com indicação bem precisa nos casos em que há formação aneurismática. Já foram descritos casos de descompressão vascular do VIII par e também de secção parcial do mesmo para o alívio dos sintomas ${ }^{8}$.

No caso de nosso paciente, é evidenciada a importância da avaliação metódica do caso, com a diferenciação pelo BERA de uma patologia retrococlear, unilateral, de causa bem definida posteriormente pelo exame de imagem. Apesar de o caso não ter tido indicação cirúrgica, é importante o conhecimento da causa do zumbido para o esclarecimento do paciente e do próprio médico, que tem a partir daí a orientação precisa para o seguimento do caso.

\section{COMENTÁRIOS FINAIS}

Este caso clínico ilustra a diversidade de causas que podem gerar o sintoma zumbido. Mesmo um quadro caracteristicamente retrococlear pode originar-se de uma patologia caracterizada por variação anatômica do normal, de evolução benigna. É de suma importância que os profissionais que tratam desse tipo de paciente tenham sempre a noção de que o zumbido não é uma doença, e sim um sintoma, e que a patologia de base deve ser exaustivamente pesquisada, para melhor condução dos casos.

\section{REFERÊNCIAS BIBLIOGRÁFICAS}

1. Schulman A. Introduction: Definition and Classification of Tinnitus. In Kitahara M. (ed): Tinnitus - Pathophysiology and Management. New York: Ikagu-Shoin; 1988, p. 1-6.

2. Hallam RS, Rachman S, Hinchecliff R. Psychological aspects of tinnitus. In Rachman R. (ed). Contributions to Medical Psychology. Oxford: Pergamon Press; 1984. p.31-4.

3. Seidman MD, Jacobson GP. Update on tinnitus. Otolaryngol Clin North Am 1996; 29(3): 455-65.

4. Lemaire MC, Beutter P. Brainstem Auditory Evoked Responses in Patientes with Tinnitus. Audiology 1995; 34: 287-300.

5. Otterstedde CR, Tischendorf M, Reisser C. Megadolichobasilarartery as the etiology in sensorineural deafness in diferential sudden deafness diagnosis: HNO 1999; 47(5): 494-6.

6. Guiral H, Risco J, Ferrer F. Otoneurological manifestations of basilar dolicoectasia - a report of six cases: Acta Otorrinolaringol Esp 1997; 48(5): 337-40.

7. Benecke JE Jr, Hitselberger WE. Vertigo caused by basilar artery compression of the VIII nerve: Laryngoscope 1988; 98 (8 pt 1): 807-9.

8. Meyerhoff WL, Mickey BE. Vascular decompression of the cochlear nerve: Laryngoscope 1988; 98: 602-4. 